\title{
On dual mixed quermassintegral quotient functions
}

Ping Zhang, Weidong Wang and Xiaohua Zhang*

"Correspondence: zhangxiaohua07@163.com

Department of Mathematics, China Three Gorges University, Yichang, 443002, P.R. China

\begin{abstract}
We introduce the notion of dual mixed quermassintegral quotient functions and establish the Brunn-Minkowski inequalities for them in this paper.
\end{abstract}

MSC: 52A20; 52A40

Keywords: dual quermassintegral; dual mixed quermassintegral quotient function; Brunn-Minkowski inequality

\section{Introduction and main results}

The setting for this paper is Euclidean $n$-space $\mathbb{R}^{n}$. Let $\mathcal{S}_{o}^{n}$ denote the set of star bodies containing the origin in their interiors in $\mathbb{R}^{n}$. Let $S^{n-1}$ denote the unit sphere in $\mathbb{R}^{n}$, and let $V(K)$ denote the $n$-dimensional volume of body $K$. For the standard unit ball $B$ in $\mathbb{R}^{n}$, we use $\omega_{n}=V(B)$ to denote its volume.

In 1975, Lutwak (see [1]) gave the notion of dual mixed volumes as follows: For $K_{1}, K_{2}, \ldots, K_{n} \in \mathcal{S}_{o}^{n}$, the dual mixed volume, $\widetilde{V}\left(K_{1}, K_{2}, \ldots, K_{n}\right)$, of $K_{1}, K_{2}, \ldots, K_{n}$ is defined by

$$
\widetilde{V}\left(K_{1}, \ldots, K_{n}\right)=\frac{1}{n} \int_{S^{n-1}} \rho\left(K_{1}, u\right) \cdots \rho\left(K_{n}, u\right) d S(u) .
$$

Taking $K_{1}=\cdots=K_{n-i}=K, K_{n-i+1}=\cdots=K_{n}=L$ in (1.1), we write $\widetilde{V}_{i}(K, L)=\widetilde{V}(K, n-$ $i ; L, i)$, where $K$ appears $n-i$ times and $L$ appears $i$ times. Then

$$
\widetilde{V}_{i}(K, L)=\frac{1}{n} \int_{S^{n-1}} \rho(K, u)^{n-i} \rho(L, u)^{i} d S(u) .
$$

Let $L=B$ in (1.2) and notice $\rho(B, \cdot)=1$, and allow $i$ is any real, then the dual quermassintegrals can be defined as follows: For $K \in \mathcal{S}_{o}^{n}$ and $i$ is any real, the dual quermassintegrals, $\widetilde{W}_{i}(K)$, of $K$ are given by (see [1])

$$
\widetilde{W}_{i}(K)=\frac{1}{n} \int_{S^{n-1}} \rho(K, u)^{n-i} d S(u) .
$$

Associated with dual quermassintegrals, Zhao (see [2]) defined the dual quermassintegral quotient functions of a star body $K$ by

$$
Q_{\widetilde{W}_{i, j}(K)}=\frac{\widetilde{W}_{i}(K)}{\widetilde{W}_{j}(K)} \quad(i, j \in \mathbb{R}) .
$$

(c) 2015 Zhang et al. This article is distributed under the terms of the Creative Commons Attribution 4.0 International License (http://creativecommons.org/licenses/by/4.0/), which permits unrestricted use, distribution, and reproduction in any medium, provided you give appropriate credit to the original author(s) and the source, provide a link to the Creative Commons license, and indicate if changes were made. 
Further, in [2] the Brunn-Minkowski type inequalities for the dual quermassintegral quotient functions of star bodies were established as follows.

Theorem A If $K, L \in \mathcal{S}_{o}^{n}$ and reals $i, j$ satisfy $i \leq n-1 \leq j \leq n$, then

$$
Q_{\widetilde{W}_{i, j}(K \tilde{+} L)}^{\frac{1}{j-i}} \leq Q_{\widetilde{W}_{i, j}(K)}^{\frac{1}{j-i}}+Q_{\widetilde{W}_{i, j}(L)}^{\frac{1}{j-i}} .
$$

Here $\tilde{+}$ is the radial Minkowski sum.

Theorem B If $K, L \in \mathcal{S}_{o}^{n}$ and reals $i, j$ satisfy $i \leq 1 \leq j \leq n$, then

$$
Q_{\widetilde{W}_{i, j}(K \check{+}+L)}^{\frac{n-1}{j-i}} \leq Q_{\widetilde{W}_{i, j}(K)}^{\frac{n-1}{j-i}}+Q_{\widetilde{W}_{i, j}(L)}^{\frac{n-1}{j-i}} .
$$

Here $\breve{+}$ is the radial Blaschke sum.

Theorem C If $K, L \in \mathcal{S}_{o}^{n}$ and reals $i, j$ satisfy $i \leq-1 \leq j \leq n$, then

$$
\frac{Q_{\widetilde{W}_{i, j}(K \hat{+} L)}^{\frac{n+1}{j-i}}}{V(K \hat{+} L)} \leq \frac{Q_{\widetilde{W}_{i, j}(K)}^{\frac{n+1}{j-i}}}{V(K)}+\frac{Q_{\widetilde{W}_{i, j}(L)}^{\frac{n+1}{j-i}}}{V(L)} .
$$

Here $\hat{+}$ is the harmonic Blaschke sum.

Motivated by the work of Zhao, we give the following definition of dual mixed quermassintegral quotient function.

Let $K_{1}=\cdots=K_{n-i-1}=K, K_{n-i}=\cdots=K_{n-1}=B, K_{n}=L$ in (1.1), then we write $\widetilde{W}_{i}(K, L)=$ $\widetilde{V}(K, n-i-1 ; B, i ; L, 1)$, where $K$ appears $n-i-1$ times, $B$ appears $i$ times and $L$ appears 1 time. Here, we allow $i$ to be any real and define as follows: For $K, L \in \mathcal{S}_{o}^{n}$ and $i$ any real, the dual mixed quermassintegrals, $\widetilde{W}_{i}(K, L)$, of $K$ and $L$ are given by

$$
\widetilde{W}_{i}(K, L)=\frac{1}{n} \int_{S^{n-1}} \rho(K, u)^{n-i-1} \rho(L, u) d S(u) .
$$

Obviously, from (1.3) and (1.5), we have $\widetilde{W}_{i}(K, K)=\widetilde{W}_{i}(K)$. According to (1.5), we define the following.

Definition 1.1 Let $K, L \in \mathcal{S}_{o}^{n}$ and $i, j \in \mathbb{R}$, the dual mixed quermassintegral quotient function, $Q_{\widetilde{W}_{i, j}(K, L)}$, of $K$ and $L$ can be defined by

$$
Q_{\widetilde{W}_{i, j}(K, L)}=\frac{\widetilde{W}_{i}(K, L)}{\widetilde{W}_{j}(K, L)} .
$$

Obviously, if $L=K$, then (1.6) is just (1.4).

The aim of this paper is to establish the following Brunn-Minkowski type inequalities for dual mixed quermassintegral quotient functions of star bodies.

Theorem 1.1 For $K, K^{\prime}, L \in \mathcal{S}_{o}^{n}$, if $i \leq n-2 \leq j<n-1$, then

$$
Q_{\widetilde{W}_{i, j}\left(K \tilde{+} K^{\prime}, L\right)}^{\frac{1}{j-i}} \leq Q_{\widetilde{W}_{i, j}(K, L)}^{\frac{1}{j-i}}+Q_{\widetilde{W}_{i, j}\left(K^{\prime}, L\right)}^{\frac{1}{j-i}}
$$


if $n-2 \leq i<n-1<j$, then

$$
Q_{\widetilde{W}_{i, j}\left(K \tilde{+} K^{\prime}, L\right)}^{\frac{1}{j-i}} \geq Q_{\widetilde{W}_{i, j}(K, L)}^{\frac{1}{j-i}}+Q_{\widetilde{W}_{i, j}\left(K^{\prime}, L\right)}^{\frac{1}{j-i}}
$$

In each case, equality holds if and only if $K$ and $K^{\prime}$ are dilates. Here $\tilde{+}$ is the radial Minkowski sum.

Theorem 1.2 For $K, K^{\prime}, L \in \mathcal{S}_{o}^{n}$, if $i \leq 0 \leq j<n-1$, then

$$
Q_{\widetilde{W}_{i, j}\left(K \breve{+} K^{\prime}, L\right)}^{\frac{n-1}{j-i}} \leq Q_{\widetilde{W}_{i, j}(K, L)}^{\frac{n-1}{j-i}}+Q_{\widetilde{W}_{i, j}\left(K^{\prime}, L\right)}^{\frac{n-1}{j-i}} ;
$$

if $0 \leq i<n-1<j$, then

$$
Q_{\widetilde{W}_{i, j}\left(K \breve{+} K^{\prime}, L\right)}^{\frac{n-1}{j-i}} \geq Q_{\widetilde{W}_{i, j}(K, L)}^{\frac{n-1}{j-i}}+Q_{\widetilde{W}_{i, j}\left(K^{\prime}, L\right)}^{\frac{n-1}{j-i}}
$$

In each case, equality holds if and only if $K$ and $K^{\prime}$ are dilates. Here $\breve{+}$ is the radial Blaschke sum.

Theorem 1.3 For $K, K^{\prime}, L \in \mathcal{S}_{o}^{n}$, if $i \leq-2 \leq j<n-1$, then

$$
\frac{Q_{\widetilde{W}_{i, j}\left(K \hat{+} K^{\prime}, L\right)}^{\frac{n+1}{j-i}}}{V\left(K \hat{+} K^{\prime}\right)} \leq \frac{Q_{\widetilde{W}_{i, j}(K, L)}^{\frac{n+1}{j-i}}}{V(K)}+\frac{Q_{\widetilde{W}_{i, j}}^{\frac{n+1}{j-i}}}{V\left(K^{\prime}\right)}
$$

if $-2 \leq i<n-1<j$, then

$$
\frac{Q_{\widetilde{W}_{i, j}\left(K \hat{+} K^{\prime}, L\right)}^{\frac{n+1}{j j i}}}{V\left(K \hat{+} K^{\prime}\right)} \geq \frac{Q_{\widetilde{W}_{i, j}(K, L)}^{\frac{n+1}{j-i}}}{V(K)}+\frac{Q_{\widetilde{W}_{i, j}\left(K^{\prime}, L\right)}^{\frac{n+1}{j-i}}}{V\left(K^{\prime}\right)} .
$$

In each case, equality holds if and only if $K$ and $K^{\prime}$ are dilates. Here $\hat{+}$ is the harmonic Blaschke sum.

\section{Preliminaries}

For a compact set $K$ in $\mathbb{R}^{n}$ which is star shaped with respect to the origin, we define the radial function $\rho_{K}(u)=\rho(K, u)$ of $K$ by

$$
\rho(K, u)=\max \{\lambda \geq 0: \lambda u \in K\}, \quad u \in S^{n-1} .
$$

If $\rho_{K}$ is positive and continuous, $K$ will be called a star body (about the origin). Two star bodies $K$ and $L$ are said to be dilates (of one another) if $\rho_{K}(u) / \rho_{L}(u)$ is independent of $u \in S^{n-1}$.

For $K_{1}, K_{2} \in \mathcal{S}_{o}^{n}$, and $\lambda_{1}, \lambda_{2} \geq 0$ (not both 0 ), the radial function of the radial Minkowski linear combination $\lambda_{1} K_{1} \tilde{+} \lambda_{2} K_{2}$ is given by Zhang (see [3]):

$$
\rho\left(\lambda_{1} K_{1} \tilde{+} \lambda_{2} K_{2}, u\right)=\lambda_{1} \rho\left(K_{1}, u\right)+\lambda_{2} \rho\left(K_{2}, u\right) .
$$


For $K_{1}, K_{2} \in \mathcal{S}_{o}^{n}$, and $\lambda_{1}, \lambda_{2} \geq 0$ (not both 0 ), the radial Blaschke linear combination $\lambda_{1}$. $K_{1} \check{+} \lambda_{2} \cdot K_{2}$ is a star body whose radial function is given by Lutwak (see [4]):

$$
\rho\left(\lambda_{1} \cdot K_{1} \check{+} \lambda_{2} \cdot K_{2}, u\right)^{n-1}=\lambda_{1} \rho\left(K_{1}, u\right)^{n-1}+\lambda_{2} \rho\left(K_{2}, u\right)^{n-1} .
$$

For $K_{1}, K_{2} \in \mathcal{S}_{o}^{n}$, and $\lambda_{1}, \lambda_{2} \geq 0$ (not both 0 ), the harmonic Blaschke linear combination $\lambda_{1} \circ K_{1} \hat{+} \lambda_{2} \circ K_{2}$ is a star body whose radial function is given by Lutwak (see [5]):

$$
\frac{\rho\left(\lambda_{1} \circ K_{1} \hat{+} \lambda_{2} \circ K_{2}, u\right)^{n+1}}{V\left(\lambda_{1} \circ K_{1} \hat{+} \lambda_{2} \circ K_{2}\right)}=\lambda_{1} \frac{\rho\left(K_{1}, u\right)^{n+1}}{V\left(K_{1}\right)}+\lambda_{2} \frac{\rho\left(K_{2}, u\right)^{n+1}}{V\left(K_{2}\right)} .
$$

\section{Proofs of theorems}

According to a generalization of the Dresher inequality (see [6]), we get the reverse Dresher inequality.

Lemma 3.1 (Dresher's inequality) Let functions $f_{1}, f_{2}, g_{1}, g_{2} \geq 0$, E is a bounded measurable subset in $\mathbb{R}^{n}$. If $p \geq 1 \geq r \geq 0$, then

$$
\left(\frac{\int_{E}\left(f_{1}+f_{2}\right)^{p} d x}{\int_{E}\left(g_{1}+g_{2}\right)^{r} d x}\right)^{\frac{1}{p-r}} \leq\left(\frac{\int_{E} f_{1}^{p} d x}{\int_{E} g_{1}^{r} d x}\right)^{\frac{1}{p-r}}+\left(\frac{\int_{E} f_{2}^{p} d x}{\int_{E} g_{2}^{r} d x}\right)^{\frac{1}{p-r}}
$$

equality holds if and only if $f_{1} / f_{2}=g_{1} / g_{2}$.

Lemma 3.2 (Reverse Dresher's inequality) Let functions $f_{1}, f_{2}, g_{1}, g_{2} \geq 0$, E is a bounded measurable subset in $\mathbb{R}^{n}$. If $1 \geq p>0>r$, then

$$
\left(\frac{\int_{E}\left(f_{1}+f_{2}\right)^{p} d x}{\int_{E}\left(g_{1}+g_{2}\right)^{r} d x}\right)^{\frac{1}{p-r}} \geq\left(\frac{\int_{E} f_{1}^{p} d x}{\int_{E} g_{1}^{r} d x}\right)^{\frac{1}{p-r}}+\left(\frac{\int_{E} f_{2}^{p} d x}{\int_{E} g_{2}^{r} d x}\right)^{\frac{1}{p-r}}
$$

equality holds if and only if $f_{1} / f_{2}=g_{1} / g_{2}$.

Proof of Lemma 3.2 If $f_{1}, f_{2}, g_{1}, g_{2} \geq 0$, and $1 \geq p>0>r$, according to the Minkowski inequality,

$$
\begin{aligned}
& \left(\int_{E}\left(f_{1}+f_{2}\right)^{p} d x\right)^{\frac{1}{p}} \geq\left(\int_{E} f_{1}^{p} d x\right)^{\frac{1}{p}}+\left(\int_{E} f_{2}^{p} d x\right)^{\frac{1}{p}}, \\
& \left(\int_{E}\left(g_{1}+g_{2}\right)^{r} d x\right)^{\frac{1}{r}} \geq\left(\int_{E} g_{1}^{r} d x\right)^{\frac{1}{r}}+\left(\int_{E} g_{2}^{r} d x\right)^{\frac{1}{r}} .
\end{aligned}
$$

For $1 \geq p>0>r$, we have

$$
\begin{aligned}
& \int_{E}\left(f_{1}+f_{2}\right)^{p} d x \geq\left(\left(\int_{E} f_{1}^{p} d x\right)^{\frac{1}{p}}+\left(\int_{E} f_{2}^{p} d x\right)^{\frac{1}{p}}\right)^{p}, \\
& \int_{E}\left(g_{1}+g_{2}\right)^{r} d x \leq\left(\left(\int_{E} g_{1}^{r} d x\right)^{\frac{1}{r}}+\left(\int_{E} g_{2}^{r} d x\right)^{\frac{1}{r}}\right)^{r} .
\end{aligned}
$$


According to the Hölder inequality, $\frac{p-r}{p}>1$, and (3.3), (3.4),

$$
\begin{aligned}
& \left(\frac{\int_{E}\left(f_{1}+f_{2}\right)^{p} d x}{\int_{E}\left(g_{1}+g_{2}\right)^{r} d x}\right)^{\frac{1}{p-r}} \geq\left[\frac{\left(\left(\int_{E} f_{1}^{p} d x\right)^{\frac{1}{p}}+\left(\int_{E} f_{2}^{p} d x\right)^{\frac{1}{p}}\right)^{p}}{\left(\left(\int_{E} g_{1}^{r} d x\right)^{\frac{1}{r}}+\left(\int_{E} g_{2}^{r} d x\right)^{\frac{1}{r}}\right)^{r}}\right]^{\frac{1}{p-r}} \\
& =\frac{\left[\left(\int_{E} f_{1}^{p} d x\right)^{\frac{1}{p}}+\left(\int_{E} f_{2}^{p} d x\right)^{\frac{1}{p}}\right]^{\frac{p}{p-r}}}{\left[\left(\int_{E} g_{1}^{r} d x\right)^{\frac{1}{r}}+\left(\int_{E} g_{2}^{r} d x\right)^{\frac{1}{r}}\right]^{\frac{r}{p-r}}} \\
& =\left[\left(\left(\int_{E} f_{1}^{p} d x\right)^{\frac{1}{p-r}}\right)^{\frac{p-r}{p}}+\left(\left(\int_{E} f_{2}^{p} d x\right)^{\frac{1}{p-r}}\right)^{\frac{p-r}{p}}\right]^{\frac{p}{p-r}} \\
& \times\left[\left(\left(\int_{E} g_{1}^{r} d x\right)^{\frac{-1}{p-r}}\right)^{\frac{-(p-r)}{r}}+\left(\left(\int_{E} g_{2}^{r} d x\right)^{\frac{-1}{p-r}}\right)^{\frac{-(p-r)}{r}}\right]^{\frac{-r}{p-r}} \\
& \geq\left(\int_{E} f_{1}^{p} d x\right)^{\frac{1}{p-r}}\left(\int_{E} g_{1}^{r} d x\right)^{\frac{-1}{p-r}}+\left(\int_{E} f_{2}^{p} d x\right)^{\frac{1}{p-r}}\left(\int_{E} g_{2}^{r} d x\right)^{\frac{-1}{p-r}} \\
& =\left(\frac{\int_{E} f_{1}^{p} d x}{\int_{E} g_{1}^{r} d x}\right)^{\frac{1}{p-r}}+\left(\frac{\int_{E} f_{2}^{p} d x}{\int_{E} g_{2}^{r} d x}\right)^{\frac{1}{p-r}} .
\end{aligned}
$$

According to the equality condition of the Minkowski inequality and the Hölder inequality, equality holds in (3.2) if and only if $f_{1} / f_{2}=g_{1} / g_{2}$.

Proof of Theorem 1.1 From (2.1), for $K, K^{\prime}, L \in \mathcal{S}_{o}^{n}$,

$$
\begin{aligned}
\widetilde{W}_{n-p-1}\left(K \tilde{+} K^{\prime}, L\right) & =\frac{1}{n} \int_{S^{n-1}} \rho_{K \tilde{+} K^{\prime}}^{p}(u) \rho_{L}(u) d S(u) \\
& =\frac{1}{n} \int_{S^{n-1}}\left(\rho_{K}(u)+\rho_{K^{\prime}}(u)\right)^{p} \rho_{L}(u) d S(u) \\
& =\frac{1}{n} \int_{S^{n-1}}\left(\rho_{K}(u) \rho_{L}^{\frac{1}{p}}(u)+\rho_{K^{\prime}}(u) \rho_{L}^{\frac{1}{p}}(u)\right)^{p} d S(u)
\end{aligned}
$$

and

$$
\widetilde{W}_{n-r-1}\left(K \tilde{+} K^{\prime}, L\right)=\frac{1}{n} \int_{S^{n-1}}\left(\rho_{K}(u) \rho_{L}^{\frac{1}{r}}(u)+\rho_{K^{\prime}}(u) \rho_{L}^{\frac{1}{r}}(u)\right)^{r} d S(u) .
$$

From (3.1), (3.5), and (3.6), for $p \geq 1 \geq r>0$, we have

$$
\begin{aligned}
\left(\frac{\widetilde{W}_{n-p-1}\left(K \tilde{+} K^{\prime}, L\right)}{\widetilde{W}_{n-r-1}\left(K \tilde{+} K^{\prime}, L\right)}\right)^{\frac{1}{p-r}}= & \left(\frac{\int_{S^{n-1}}\left(\rho_{K}(u) \rho_{L}^{\frac{1}{p}}(u)+\rho_{K^{\prime}}(u) \rho_{L}^{\frac{1}{p}}(u)\right)^{p} d S(u)}{\int_{S^{n-1}}\left(\rho_{K}(u) \rho_{L}^{\frac{1}{r}}(u)+\rho_{K^{\prime}}(u) \rho_{L}^{\frac{1}{r}}(u)\right)^{r} d S(u)}\right)^{\frac{1}{p-r}} \\
\leq & \left(\frac{\int_{S^{n-1}}\left(\rho_{K}(u) \rho_{L}^{\frac{1}{p}}(u)\right)^{p} d S(u)}{\int_{S^{n-1}}\left(\rho_{K}(u) \rho_{L}^{\frac{1}{r}}(u)\right)^{r} d S(u)}\right)^{\frac{1}{p-r}} \\
& +\left(\frac{\int_{S^{n-1}}\left(\rho_{K^{\prime}}(u) \rho_{L}^{\frac{1}{p}}(u)\right)^{p} d S(u)}{\int_{S^{n-1}}\left(\rho_{K^{\prime}}(u) \rho_{L}^{\frac{1}{r}}(u)\right)^{r} d S(u)}\right)^{\frac{1}{p-r}} \\
= & \left(\frac{\int_{S^{n-1}} \rho_{K}^{p}(u) \rho_{L}(u) d S(u)}{\int_{S^{n-1}} \rho_{K}^{r}(u) \rho_{L}(u) d S(u)}\right)^{\frac{1}{p-r}}
\end{aligned}
$$




$$
\begin{aligned}
& +\left(\frac{\int_{S^{n-1}} \rho_{K^{\prime}}^{p}(u) \rho_{L}(u) d S(u)}{\int_{S^{n-1}} \rho_{K^{\prime}}^{r}(u) \rho_{L}(u) d S(u)}\right)^{\frac{1}{p-r}} \\
= & \left(\frac{\widetilde{W}_{n-p-1}(K, L)}{\widetilde{W}_{n-r-1}(K, L)}\right)^{\frac{1}{p-r}}+\left(\frac{\widetilde{W}_{n-p-1}\left(K^{\prime}, L\right)}{\widetilde{W}_{n-r-1}\left(K^{\prime}, L\right)}\right)^{\frac{1}{p-r}} .
\end{aligned}
$$

According to the equality condition of inequality (3.1), we see that equality holds in (3.7) if and only if $K$ and $L, K^{\prime}$, and $L$ are dilates, respectively. So $K$ and $K^{\prime}$ are dilates.

Let $i=n-p-1, j=n-r-1$, then $p \geq 1 \geq r>0$ and $i \leq n-2 \leq j<n-1$ are equivalent. This and (3.7) yield inequality (1.7) and its equality condition.

Similarly, if $1 \geq p>0>r$, according to (3.2), (3.5), and (3.6), we have

$$
\left(\frac{\widetilde{W}_{n-p-1}\left(K \tilde{+} K^{\prime}, L\right)}{\widetilde{W}_{n-r-1}\left(K \tilde{+} K^{\prime}, L\right)}\right)^{\frac{1}{p-r}} \geq\left(\frac{\widetilde{W}_{n-p-1}(K, L)}{\widetilde{W}_{n-r-1}(K, L)}\right)^{\frac{1}{p-r}}+\left(\frac{\widetilde{W}_{n-p-1}\left(K^{\prime}, L\right)}{\widetilde{W}_{n-r-1}\left(K^{\prime}, L\right)}\right)^{\frac{1}{p-r}},
$$

and equality holds if and only if $K$ and $K^{\prime}$ are dilates.

Let $i=n-p-1, j=n-r-1$, then (3.8) gives inequality (1.8) and its equality condition.

Proof of Theorem 1.2 From (2.2), for $K, K^{\prime}, L \in \mathcal{S}_{o}^{n}$, we have

$$
\begin{aligned}
\widetilde{W}_{n-p-1}\left(K \breve{+} K^{\prime}, L\right) & =\frac{1}{n} \int_{S^{n-1}} \rho_{K \breve{+} K^{\prime}}^{p}(u) \rho_{L}(u) d S(u) \\
& =\frac{1}{n} \int_{S^{n-1}}\left(\rho_{K}^{n-1}(u)+\rho_{K^{\prime}}^{n-1}(u)\right)^{\frac{p}{n-1}} \rho_{L}(u) d S(u) \\
& =\frac{1}{n} \int_{S^{n-1}}\left(\rho_{K}^{n-1}(u) \rho_{L}^{\frac{n-1}{p}}(u)+\rho_{K^{\prime}}^{n-1}(u) \rho_{L}^{\frac{n-1}{p}}(u)\right)^{\frac{p}{n-1}} d S(u)
\end{aligned}
$$

and

$$
\begin{aligned}
\widetilde{W}_{n-r-1}\left(K \breve{+} K^{\prime}, L\right) & =\frac{1}{n} \int_{S^{n-1}} \rho_{K \breve{+} K^{\prime}}^{r}(u) \rho_{L}(u) d S(u) \\
& =\frac{1}{n} \int_{S^{n-1}}\left(\rho_{K}^{n-1}(u) \rho_{L}^{\frac{n-1}{r}}(u)+\rho_{K^{\prime}}^{n-1}(u) \rho_{L}^{\frac{n-1}{r}}(u)\right)^{\frac{r}{n-1}} d S(u) .
\end{aligned}
$$

According to (3.1), (3.9), and (3.10), for $p \geq n-1 \geq r>0$,

$$
\begin{aligned}
Q_{\widetilde{W}_{n-p-1, n-r-1}^{\frac{n-1}{p-r}}\left(K \breve{+} K^{\prime}, L\right)}= & {\left[\frac{\widetilde{W}_{n-p-1}\left(K \breve{+} K^{\prime}, L\right)}{\widetilde{W}_{n-r-1}\left(K \breve{+} K^{\prime}, L\right)}\right]^{\frac{n-1}{p-r}} } \\
= & {\left[\frac{\int_{S^{n-1}}\left(\rho_{K}^{n-1}(u) \rho_{L}^{\frac{n-1}{p}}(u)+\rho_{K^{\prime}}^{n-1}(u) \rho_{L}^{\frac{n-1}{p}}(u)\right)^{\frac{p}{n-1}} d S(u)}{\int_{S^{n-1}}\left(\rho_{K}^{n-1}(u) \rho_{L}^{\frac{n-1}{r}}(u)+\rho_{K^{\prime}}^{n-1}(u) \rho_{L}^{\frac{n-1}{r}}(u)\right)^{\frac{r}{n-1}} d S(u)}\right]^{\frac{n-1}{p-r}} } \\
\leq & {\left[\frac{\int_{S^{n-1}}\left(\rho_{K}^{n-1}(u) \rho_{L}^{\frac{n-1}{p}}(u)\right)^{\frac{p}{n-1}} d S(u)}{\int_{S^{n-1}}\left(\rho_{K}^{n-1}(u) \rho_{L}^{\frac{n-1}{r}}(u)\right)^{\frac{r}{n-1}} d S(u)}\right]^{\frac{n-1}{p-r}} } \\
& +\left[\frac{\int_{S^{n-1}}\left(\rho_{K^{\prime}}^{n-1}(u) \rho_{L}^{\frac{n-1}{p}}(u)\right)^{\frac{p}{n-1}} d S(u)}{\int_{S^{n-1}}\left(\rho_{K^{\prime}}^{n-1}(u) \rho_{L}^{\frac{n-1}{r}}(u)\right)^{\frac{r}{n-1}} d S(u)^{\frac{n-1}{p-r}}}\right]^{\frac{n}{p}}
\end{aligned}
$$




$$
\begin{aligned}
& =\left[\frac{\int_{S^{n-1}} \rho_{K}^{p}(u) \rho_{L}(u) d S(u)}{\int_{S^{n-1}} \rho_{K}^{r}(u) \rho_{L}(u) d S(u)}\right]^{\frac{n-1}{p-r}}+\left[\frac{\int_{S^{n-1}} \rho_{K^{\prime}}^{p}(u) \rho_{L}(u) d S(u)}{\int_{S^{n-1}} \rho_{K^{\prime}}^{r}(u) \rho_{L}(u) d S(u)}\right]^{\frac{n-1}{p-r}} \\
& =\left[\frac{\widetilde{W}_{n-p-1}(K, L)}{\widetilde{W}_{n-r-1}(K, L)}\right]^{\frac{n-1}{p-r}}+\left[\frac{\widetilde{W}_{n-p-1}\left(K^{\prime}, L\right)}{\widetilde{W}_{n-r-1}\left(K^{\prime}, L\right)}\right]^{\frac{n-1}{p-r}} \\
& =Q_{\widetilde{W}_{n-p-1, n-r-1}(K, L)}^{\frac{n-1}{p-r}}+Q_{\widetilde{W}_{n-p-1, n-r-1}\left(K^{\prime}, L\right)}^{\frac{n-1}{p-r}}
\end{aligned}
$$

Then

$$
Q_{\widetilde{W}_{n-p-1, n-r-1}\left(K+K^{\prime}, L\right)}^{\frac{n-1}{p-r}} \leq Q_{\widetilde{W}_{n-p-1, n-r-1}(K, L)}^{\frac{n-1}{p-r}}+Q_{\widetilde{W}_{n-p-1, n-r-1}\left(K^{\prime}, L\right)}^{\frac{n-1}{p-r}} .
$$

According to the equality condition of inequality (3.1), we see that equality holds in (3.11) if and only if $K$ and $K^{\prime}$ are dilates.

Let $i=n-p-1$ and $j=n-r-1$, then $p \geq n-1 \geq r>0$ and $i \leq 0 \leq j<n-1$ are equivalent. This and (3.11) yield inequality (1.9) and its equality condition.

Similarly, if $n-1 \geq p>0>r$, according to (3.2), (3.9), and (3.10), we have

$$
\left(\frac{\widetilde{W}_{n-p-1}\left(K \tilde{+} K^{\prime}, L\right)}{\widetilde{W}_{n-r-1}\left(K \tilde{+} K^{\prime}, L\right)}\right)^{\frac{1}{p-r}} \geq\left(\frac{\widetilde{W}_{n-p-1}(K, L)}{\widetilde{W}_{n-r-1}(K, L)}\right)^{\frac{1}{p-r}}+\left(\frac{\widetilde{W}_{n-p-1}\left(K^{\prime}, L\right)}{\widetilde{W}_{n-r-1}\left(K^{\prime}, L\right)}\right)^{\frac{1}{p-r}},
$$

and equality holds if and only if $K$ and $K^{\prime}$ are dilates.

Let $i=n-p-1$ and $j=n-r-1$, then (3.12) gives inequality (1.10) and its equality condition.

Proof of Theorem 1.3 From (2.3), for $K, K^{\prime}, L \in \mathcal{S}_{o}^{n}$,

$$
\begin{aligned}
\frac{\widetilde{W}_{n-p-1}\left(K \hat{+} K^{\prime}, L\right)}{V\left(K \hat{+} K^{\prime}\right)^{p /(n+1)}} & =\frac{1}{n} \int_{S^{n-1}} \frac{\rho_{K \hat{+} K^{\prime}}^{p}(u) \rho_{L}(u)}{V\left(K \hat{+} K^{\prime}\right)^{p /(n+1)}} d S(u) \\
& =\frac{1}{n} \int_{S^{n-1}}\left(\frac{\rho_{K+K^{\prime}}^{n+1}(u)}{V\left(K \hat{+} K^{\prime}\right)}\right)^{\frac{p}{n+1}} \rho_{L}(u) d S(u) \\
& =\frac{1}{n} \int_{S^{n-1}}\left(\frac{\rho_{K}^{n+1}(u)}{V(K)}+\frac{\rho_{K^{\prime}}^{n+1}(u)}{V\left(K^{\prime}\right)}\right)^{\frac{p}{n+1}} \rho_{L}(u) d S(u) \\
& =\frac{1}{n} \int_{S^{n-1}}\left(\frac{\rho_{K}^{n+1}(u) \rho_{L}^{\frac{n+1}{p}}(u)}{V(K)}+\frac{\rho_{K^{\prime}}^{n+1}(u) \rho_{L}^{\frac{n+1}{p}}(u)}{V\left(K^{\prime}\right)}\right)^{\frac{p}{n+1}} d S(u)
\end{aligned}
$$

and

$$
\frac{\widetilde{W}_{n-r-1}\left(K \hat{+} K^{\prime}, L\right)}{V\left(K \hat{+} K^{\prime}\right)^{r /(n+1)}}=\frac{1}{n} \int_{S^{n-1}}\left(\frac{\rho_{K}^{n+1}(u) \rho_{L}^{\frac{n+1}{r}}(u)}{V(K)}+\frac{\rho_{K^{\prime}}^{n+1}(u) \rho_{L}^{\frac{n+1}{r}}(u)}{V\left(K^{\prime}\right)}\right)^{\frac{r}{n+1}} d S(u) .
$$

According to (3.1), (3.13), and (3.14), for $p \geq n+1>r>0$,

$$
\begin{aligned}
Q_{\widetilde{W}_{n-p-1, n-r-1}\left(K \hat{+} K^{\prime}, L\right)}^{\frac{n+1}{p-r}} & =\left(\frac{\widetilde{W}_{n-p-1}\left(K \hat{+} K^{\prime}, L\right)}{\widetilde{W}_{n-r-1}\left(K \hat{+} K^{\prime}, L\right)}\right)^{\frac{n+1}{p-r}} \\
& =V\left(K \hat{+} K^{\prime}\right)\left[\frac{\int_{S^{n-1}}\left(\frac{\rho_{K}^{n+1}(u)}{V(K)} \rho_{L}^{\frac{n+1}{p}}(u)+\frac{\rho_{K^{\prime}}^{n+1}(u)}{V\left(K^{\prime}\right)} \rho_{L}^{\frac{n+1}{p}}(u)\right)^{\frac{p}{n+1}} d S(u)}{\int_{S^{n-1}}\left(\frac{\rho_{K}^{n+1}(u)}{V(K)} \rho_{L}^{\frac{n+1}{r}}(u)+\frac{\rho_{K^{\prime}}^{n+1}(u)}{V\left(K^{\prime}\right)} \rho_{L}^{\frac{n+1}{r}}(u)\right)^{\frac{r}{n+1}} d S(u)}\right]^{\frac{n+1}{p-r}}
\end{aligned}
$$




$$
\begin{aligned}
& \leq V\left(K \hat{+} K^{\prime}\right)\left[\frac{\int_{S^{n-1}}\left(V(K)^{-1} \rho_{K}^{n+1}(u) \rho_{L}^{\frac{n+1}{p}}(u)\right)^{\frac{p}{n+1}} d S(u)}{\int_{S^{n-1}}\left(V(K)^{-1} \rho_{K}^{n+1}(u) \rho_{L}^{\frac{n+1}{r}}(u)\right)^{\frac{r}{n+1}} d S(u)}\right]^{\frac{n+1}{p-r}} \\
& +V\left(K \hat{+} K^{\prime}\right)\left[\frac{\int_{S^{n-1}}\left(V\left(K^{\prime}\right)^{-1} \rho_{K^{\prime}}^{n+1}(u) \rho_{L}^{\frac{n+1}{p}}(u)\right)^{\frac{p}{n+1}} d S(u)}{\int_{S^{n-1}}\left(V\left(K^{\prime}\right)^{-1} \rho_{K^{\prime}}^{n+1}(u) \rho_{L}^{\frac{n+1}{r}}(u)\right)^{\frac{r}{n+1}} d S(u)}\right]^{\frac{n+1}{p-r}} \\
& =V\left(K \hat{+} K^{\prime}\right)\left[\frac{\int_{S^{n-1}}\left(V(K)^{-1}\right)^{\frac{p}{n+1}} \rho_{K}^{p}(u) \rho_{L}(u) d S(u)}{\int_{S^{n-1}}\left(V(K)^{-1}\right)^{\frac{r}{n+1}} \rho_{K}^{r}(u) \rho_{L}(u) d S(u)}\right]^{\frac{n+1}{p-r}} \\
& +V\left(K \hat{+} K^{\prime}\right)\left[\frac{\int_{S^{n-1}}\left(V\left(K^{\prime}\right)^{-1}\right)^{\frac{p}{n+1}} \rho_{K^{\prime}}^{p}(u) \rho_{L}(u) d S(u)}{\int_{S^{n-1}}\left(V\left(K^{\prime}\right)^{-1}\right)^{\frac{r}{n+1}} \rho_{K^{\prime}}^{r}(u) \rho_{L}(u) d S(u)}\right]^{\frac{n+1}{p-r}} \\
& =\frac{V\left(K \hat{+} K^{\prime}\right)}{V(K)}\left(\frac{\int_{S^{n-1}} \rho_{K}^{p}(u) \rho_{L}(u) d S(u)}{\int_{S^{n-1}} \rho_{K}^{r}(u) \rho_{L}(u) d S(u)}\right)^{\frac{n+1}{p-r}} \\
& +\frac{V\left(K \hat{+} K^{\prime}\right)}{V\left(K^{\prime}\right)}\left(\frac{\int_{S^{n-1}} \rho_{K^{\prime}}^{p}(u) \rho_{L}(u) d S(u)}{\int_{S^{n-1}} \rho_{K^{\prime}}^{r}(u) \rho_{L}(u) d S(u)}\right)^{\frac{n+1}{p-r}} \\
& =\frac{V\left(K \hat{+} K^{\prime}\right)}{V(K)}\left(\frac{\widetilde{W}_{n-p-1}(K, L)}{\widetilde{W}_{n-r-1}(K, L)}\right)^{\frac{n+1}{p-r}} \\
& +\frac{V\left(K \hat{+} K^{\prime}\right)}{V\left(K^{\prime}\right)}\left(\frac{\widetilde{W}_{n-p-1}\left(K^{\prime}, L\right)}{\widetilde{W}_{n-r-1}\left(K^{\prime}, L\right)}\right)^{\frac{n+1}{p-r}}
\end{aligned}
$$

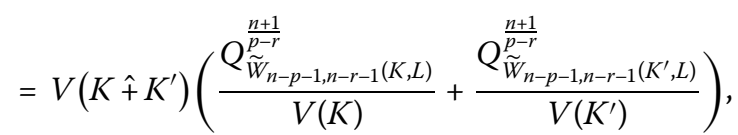

i.e.,

$$
\frac{Q_{\widetilde{W}_{n-p-1, n-r-1}\left(K \hat{+} K^{\prime}, L\right)}^{\frac{n+1}{p-r}}}{V\left(K \hat{+} K^{\prime}\right)} \leq \frac{Q_{\widetilde{W}_{n-p-1, n-r-1}(K, L)}^{\frac{n+1}{p-r}}}{V(K)}+\frac{Q_{\widetilde{W}_{n-p-1, n-r-1}\left(K^{\prime}, L\right)}^{\frac{n+1}{p-r}}}{V\left(K^{\prime}\right)} .
$$

According to the equality condition of inequality (3.1), we see that equality holds in (3.15) if and only if $K$ and $K^{\prime}$ are dilates.

Let $i=n-p-1$ and $j=n-r-1$, then $p \geq n+1 \geq r>0$ and $i \leq-2 \leq j<n-1$ are equivalent. This and (3.15) yield inequality (1.11) and its equality condition.

If $n+1 \geq p>0>r$, according to (3.2), (3.13), and (3.14), we have

$$
\frac{Q_{\widetilde{W}_{n-p-1, n-r-1}\left(K \hat{+} K^{\prime}, L\right)}^{\frac{n+1}{p-r}}}{V\left(K \hat{+} K^{\prime}\right)} \geq \frac{Q_{\widetilde{W}_{n-p-1, n-r-1}(K, L)}^{\frac{n+1}{p-r}}}{V(K)}+\frac{Q_{\widetilde{W}_{n-p-1, n-r-1}\left(K^{\prime}, L\right)}^{\frac{n+1}{p-r}}}{V\left(K^{\prime}\right)},
$$

with equality if and only if $K$ and $K^{\prime}$ are dilates.

Let $i=n-p-1$ and $j=n-r-1$, then (3.16) gives inequality (1.12) and its equality condition. 
Acknowledgements

This work was supported by the Natural Science Foundation of China (Grant Nos. 11371224 and 11102101).

Received: 25 March 2015 Accepted: 16 October 2015 Published online: 26 October 2015

\section{References}

1. Lutwak, E: Dual mixed volumes. Pac. J. Math. 58, 531-538 (1975)

2. Zhao, CJ: On volume quotient functions. Indag. Math. 24, 57-67 (2013)

3. Zhang, GY: Centered bodies and dual mixed volumes. Trans. Am. Math. Soc. 345, 777-801 (1994)

4. Lutwak, E: Intersection bodies and dual mixed volumes. Adv. Math. 71, 232-261 (1988)

5. Lutwak, E: Centroid bodies and dual mixed volumes. Proc. Lond. Math. Soc. 60, 365-391 (1990)

6. Pečarić, JE, Beeasck, PR: On Jessen's inequality for convex functions. J. Math. Anal. Appl. 118, 125-144 (1986)

\section{Submit your manuscript to a SpringerOpen ${ }^{\circ}$ journal and benefit from:}

- Convenient online submission

Rigorous peer review

- Immediate publication on acceptance

- Open access: articles freely available online

- High visibility within the field

- Retaining the copyright to your article 